\title{
Tin oxide as a transparent electrode material for light-emitting diodes fabricated with poly ( $p$-phenylene vinylene)
}

\author{
I A HÜMMELGEN*, Y P YADAVA, L S ROMAN, A C ARIAS, \\ $M$ R FERNANDES ${ }^{\dagger}$ and $F$ C NART $^{\dagger}$ \\ Departamento de Física, Universidade Federal do Paraná, Cx. Postal 19081, 81531-990 \\ Curitiba PR, Brasil \\ †'Instituto de Química de São Carlos, Universidade de São Paulo, Cx. Postal 780, 13560-970 \\ São Carlos SP, Brasil
}

\begin{abstract}
We have fabricated light emitting diodes (LED) with poly ( $p$-phenylene vinylene) (PPV) as the emissive layer and tin oxide (TO) as the transparent conducting positive electrode and aluminium as the negative electrode. The fabrication conditions are optimized for visible light emission in these TO/PPV/Al LEDs. The threshold voltage for substantial charge injection for visible light emission in these LEDs lies below $10 \mathrm{~V}$. The device fabrication and electrical characterization of TO/PPV/Al LEDs are discussed in this communication.
\end{abstract}

Keywords. Light emitting diodes; conjugated polymer; poly ( $p$-phenylene vinylene); tin oxide; aluminium; electrical characteristics.

\section{Introduction}

The recent discovery of visible light emission from conjugated polymer diodes has stimulated great research interest in these polymer materials in applications as large area thin film light emitting devices (LED) (Burroughes et al 1990; Braun and Heeger 1991; Grem et al 1992; Baigent et al 1994). Among the conjugated polymer systems poly ( $p$-phenylene vinylene) (PPV) and its derivatives are of particular importance due to their promising prospects for LEDs and since the first report of LED operation in PPV (Burroughes et al 1990), much effort has been devoted to the fabrication and characterization of ITO/PPV/metal (Al, $\mathrm{Mg}, \mathrm{Ca}$ etc.), (ITO - indium tin oxide) LED configuration using ITO as transparent positive electrode(Brown et al 1992; Karg et al 1993; Mark et al 1993; Parker 1994). To the best of our knowledge, we, for the first time, have fabricated PPV LEDs with TO/PPV/Al (TO - tin oxide) configuration using TO as the transparent positive electrode and optimized the fabrication conditions for visible light emission in these devices. The threshold for substantial charge injection for LED operation in TO/PPV/Al LEDs lies below $10 \mathrm{~V}$ at room temperature, comparable to those reported in ITO/PPV/Al LEDs at this temperature. In this communication, we describe the device fabrication and present the results of electrical characterization of TO/PPV/Al LEDs.

\section{Experimental}

The PPV films used in the present investigation were prepared via the sulphonium polyelectrolyte precursor route (Bradley 1987). The precursor was spin coated onto TO/glass substrates and subsequent thermal conversion to PPV (Antomaidis et al

\footnotetext{
* Corresponding author
} 

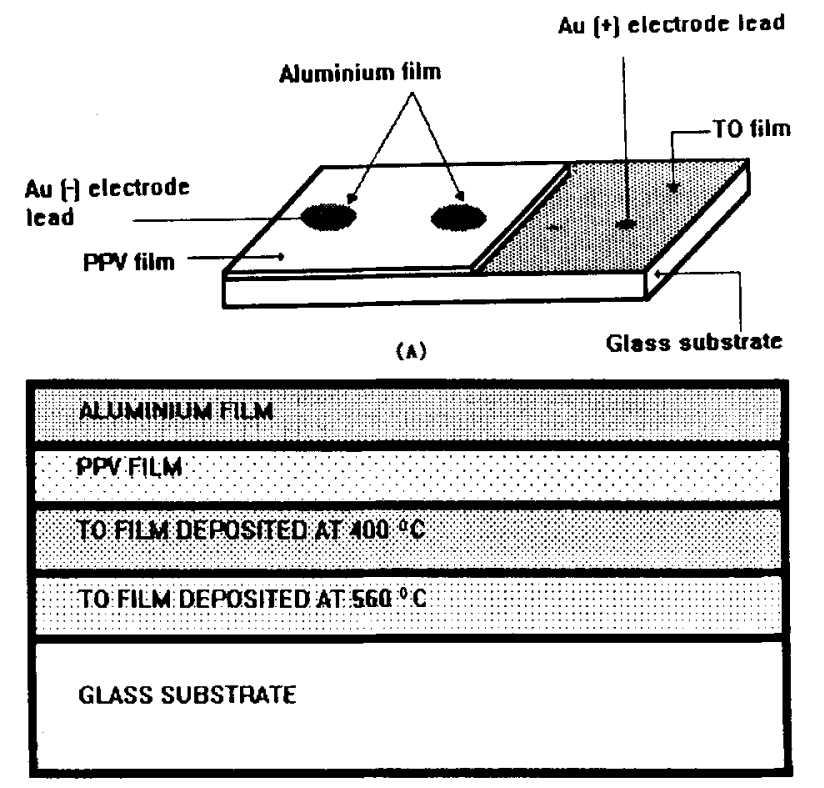

(B)

Figure 1. (A) Device structure of the TO/PPV/Al light emitting diode and (B) schematic structure of PPV, TO and Al layers of TO/PPV/Al light emitting diodes.

1994) was carried out by annealing the precursor films at $250^{\circ} \mathrm{C}$ for $2 \mathrm{~h}$ in argon atmosphere. The resulting PPV films had uniform surfaces with thickness of about $270 \mathrm{~nm}$.

The structure for LED operation was fabricated with PPV films formed on TO bottom transparent electrodes deposited on chemically cleaned glass substrates. The TO film deposition on glass substrates was done by chemical vapour deposition (CVD) using tin chloride $\left(\mathrm{SnCl}_{2} 2 \mathrm{H}_{2} \mathrm{O}\right)$ in two steps. Initially, TO films were deposited at a substrate temperature of $560^{\circ} \mathrm{C}$ and furnace cooled to room temperature, yielding the sheet resistance of $54 \mathrm{ohms}$. In the next step, we again deposited TO film over the previous TO film at a substrate temperature of $400^{\circ} \mathrm{C}$ and furnace cooled to room temperature. The final sheet resistance of the TO films was $1.2 \mathrm{k}$ ohms. The details of the TO film preparation and characterization is given in our earlier publication (Roman et al 1995). The optical transmittance of the double layer TO electrode was measured by a UV-visible spectrometer (Hewlett Packered model 8452) and the optical transmittance of this double layer TO electrode is greater than 91 percent in the visible region. The two-step TO deposition was necessary to produce visible light emission in TO/PPV/Al LEDs. Al metal contacts were deposited on top of the PPV films by vacuum evaporation at a pressure of $1 \times 10^{-5}$ Torr, yielding active areas of $9.6 \mathrm{~mm}^{2}$. The electrical connections to the $\mathrm{TO}$ and $\mathrm{Al}$ electrodes were made using fine gold wires and conducting carbon paste. The device structure of TO/PPV/Al LEDs and the systematic layered structure of TO, PPV and Al layers are shown in figure 1.

The current-voltage characteristics of the TO/PPV/Al LEDs were studied at room temperature, using a Keithley 196 System DMM, a Tektronix DM2516G programmable multimeter and a Leybold DC power supply. Figure 2 shows the current-voltage 

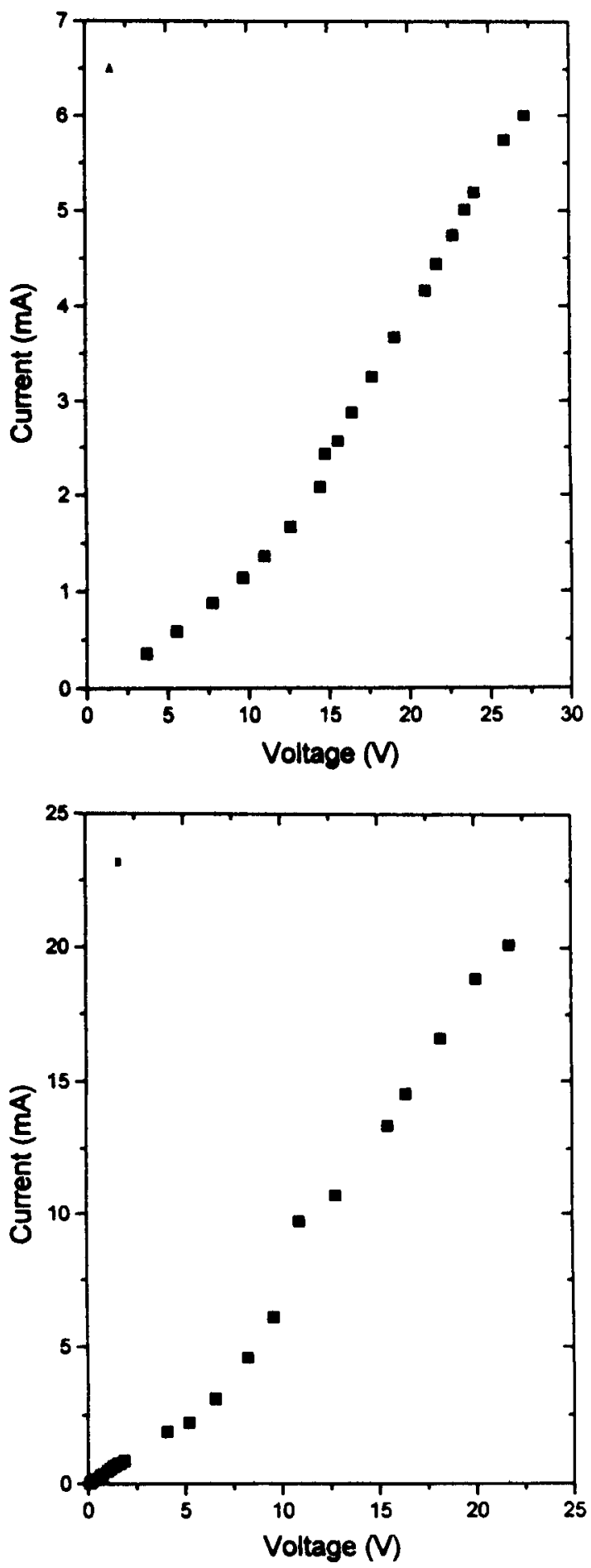

Figure 2. (A, B). Current-voltage characteristics for two typical TO/PPV/Al light emitting diodes. The thickness of PPV layer is $270 \mathrm{~nm}$ and the active area of the device is $9.6 \mathrm{~mm}^{2}$. 
characteristics of two typical TO/PPV/Al LEDs having PPV films of thickness $270 \mathrm{~nm}$ and active area of $9.6 \mathrm{~mm}^{2}$. The threshold for substantial charge injection for visible light emission in these devices lies below $10 \mathrm{~V}$ at room temperature and in forward bias visible light is emitted above $5 \mathrm{~V}$. In these LEDs the TO contacts consist of two layers. The first one deposited onto the glass substrate at $560^{\circ} \mathrm{C}$ presents low electrical resistance (sheet resistance $54 \mathrm{ohms}$ ) in comparison to the second layer which was deposited at $400^{\circ} \mathrm{C}$ (final sheet resistance $1.2 \mathrm{k}$ ohms). The first TO layer permits one to obtain an approximately homogenous distribution of the electrical field in the PPV between TO and Al electrodes. The second TO layer has been deposited to improve the light emission characteristics of the LED. We observed that TO/PPV/Al LEDs fabricated without this second TO layer were not able to produce visible light emission.

\section{Results and discussion}

We tentatively interpret these results as follows. Considering the rigid band model, the best device performance will occur when the polymer and the electrode materials are matched such that the work function of the anode equals the ionization potential of the polymer and the work function of the cathode equals the electron affinity of the polymer (Parker 1994) i.e. for an ideal device the electrodes are matched to the LUMO and HOMO of the polymer. In non-ideal devices the light emission efficiency is controlled by field emission of both carrier types through interface barriers caused by the band offset between polymer and electrodes (Marks et al 1993; Parker 1994). The problem in this case consists in the control of charge injection and transport to ensure that the currents of electrons and holes injected at opposite electrodes are balanced, so that there is not a preponderence of one charge type that carries current from an electrode to the other without encountering the opposite charge carriers within the bulk of the polymer (Baigent et al 1994). A strategy to solve this problem is to match the barriers for electron and hole injection by selection of the work function of the electrodes. This procedure is commonly adopted, but is restricted to the change of electron injection contact, whereas ITO contact remains untouched. In the present work, we choose the other way. The electron injection contact i.e. Al has been retained whereas for positive electrode we used, double layer of TO contact. In our opinion the presence of the highly resistive (surface resistance $=1.2 \mathrm{k} \mathrm{ohms}$ ) second TO layer (discussed earlier) deposited at $400^{\circ} \mathrm{C}$ enables to realign the relative positions of the electrode Fermi energy levels and the polymer bands (Parker and Kim 1994) thereby allowing tunnelling of the charge carriers into both HOMO and LUMO of the PPV and consequently the light emission characteristics of TO/PPV/AI LEDs.

Theoretical calculations locate the HOMO and LUMO levels of PPV at $5.05 \mathrm{eV}$ and $2.73 \mathrm{eV}$ relatively to vacuum level, respectively (Bredas and Heeger 1994). Considering that the potential barrier for electron injection from $\mathrm{Al}$ to PPV is expected to be around $0.2 \mathrm{eV}$ (Marks et al 1993), it is reasonable to consider that the double layer TO electrode has its Fermi energy level at the TO/PPV interface located nearer to the value $4.85 \mathrm{eV}$ than in the case of single deposited TO layer and that this realignment improves the LED characteristics i.e. the introduction of the $400^{\circ} \mathrm{C}$ deposited TO layer operates so as to produce barrier height for electrons and holes that are approximately at the same magnitude. 
The threshold voltage for substantial light emission depends, under the scope of the rigid band model, on the thickness of the PPV layer and the barrier height for charge carrier injection. The height of both barriers limits the light emission and consequently the device efficiency. In devices which present different barrier heights for electron and hole injection, the major part of the charge current contributes only to the Joule heat generation that has bad consequences on device durability and stability.

The observations discussed in this paper open new perspectives, which are associated with the control of the band offsets of both positive (via different processing techniques) and negative electrodes and the use of chemically stable metals as electron injection contacts in conjugated polymer based LEDs. The detailed investigation on TO/PPV interface and charge transport characteristics are underway and will be reported in due course.

\section{Conclusions}

In summary, we have fabricated PPV conjugated polymer based LEDs using tin oxide as transparent positive electrode, for the first time, and studied the electrical characteristics of TO/PPV/Al devices. It is found that the threshold voltage for visible light emission in these LEDs lies below $10 \mathrm{~V}$, comparable to those of ITO/PPV/AI LEDs widely reported in literature. As the production of transparent $\mathrm{TO}$ thin film electrodes is relatively simpler and much economical than that of ITO electrodes, this opens new perspectives associated with electrodes to control the band offsets and balanced charge injection for visible light emission in conjugated polymer based light emitting diodes.

\section{Acknowledgements}

This research work is performed under a PADCT Proc. 62.0074/94 project. Financial support to authors (HIA and YYP) from CNPq and (RLS) from CAPES is gratefully acknowledged.

\section{References}

Antomaidis H, Hsiech B R, Abkowitz M A, Jenekhe S A and Stolka M 1994 Synth. Met. 62263

Baigent D R, Greenham N C, Gruner J, Marks R N, Friend R H, Moratti S C and Holmes A B 1994 Synth. Met. 673 and references therein

Bradley D D C 1987 J. Phys. D. 201383

Braun D and Heeger A J 1991 Appl. Phys. Lett. 581982

Bredas J L and Heeger A J 1994 Chem. Phys. Lett. 217507

Brown A R, Bradley D D C, Burroughes J H, Friend R H, Greenham N C, Burns P L, Holmes A B and Kraft A 1992 Appl. Phys. Lett. 612793

Burroughes J H, Bradley D D C, Brown A R, Marks R N, Mackay K, Friend R H, Burns P L and Holmes A B 1990 Nature 347539

Grem G, Leditzky G, Ullrich B and Leising G 1992 Adv. Mater. 436

Karg S, Riess W, Dyakonov V and Schwoerer M 1993 Synth. Met. 54427

Marks R N, Bradley D D C, Jackson R W, Burn P L and Holmes A B 1993 Synth. Met. 55-57 4128

Parker I D 1994 J. Appl. Phys. 751656

Parker I D and Kim H H 1994 Appl. Phys. Lett. 641774

Roman L S, Yadava Y P, Denicoló G, Arias A C and Hümmelgen I A 1995 J. Mater. Sci. Materials in Electronics (in press) 